\title{
Empagliflozin promises to bridge the gap between non-alcoholic fatty liver disease, type 2 diabetes, and cardiovascular disease
}

\author{
Dimitrios Patoulias ${ }^{1}$, Maria Kalogirou ${ }^{2}$ \\ ${ }^{1} 1^{\text {st }}$ Department of Internal Medicine, General Hospital “Hippokration”, Thessaloniki, Greece \\ ${ }^{2} 4^{\text {th }}$ Department of Internal Medicine, Aristotle University of Thessaloniki, General Hospital “Hippokration”, Thessaloniki, Greece
}

Gastroenterology Rev 2018; 13 (4): 337-339

DOI: https://doi.org/10.5114/pg.2018.79815

Address for correspondence: Dimitrios Patoulias MD, $1^{\text {st }}$ Department of Internal Medicine, General Hospital "Hippokration", 49 Konstantinoupoleos St, 54642 Thessaloniki, Greece, phone: +30 6946900777, e-mail: dipatoulias@gmail.com

Non-alcoholic fatty liver disease (NAFLD) represents the commonest chronic liver disease in the Western world, while its prevalence among patients with type 2 diabetes (T2D) is as much as 70\% [1]. Non-alcoholic fatty liver disease shares common pathophysiologic mechanisms with T2D, mainly insulin resistance, lipotoxicity, and inflammation [2].

Besides the well-established role of T2D in the development of cardiovascular disease (CVD) [3], NAFLD has also emerged as a potential predictor of CVD. Previous data support the association between NAFLD and subclinical atherosclerosis, with a remarkable higher probability of its presence among patients with NAFLD compared to controls (OR $=1.60,95 \% \mathrm{Cl}: 1.45-1.78$ ) [4]. Increased arterial stiffness [5], coronary artery calcification [6], elevated carotid intimal media thickness [7], and impaired endothelial function [8] have been previously shown in patients with NAFLD.

Targher et al. have demonstrated that patients with NAFLD exhibit almost $64 \%$ greater probability of experiencing fatal or non-fatal CVD events, compared to controls ( $O R=1.64,95 \% \mathrm{Cl}: 1.26-2.13)$ [9]. Similar results were obtained from another meta-analysis conducted by Mahfood Haddad et al., who demonstrated a significantly higher risk of clinical cardiovascular events in patients with NAFLD compared to controls up to $77 \%(\mathrm{RR}=1.77,95 \% \mathrm{Cl}: 1.26-2.48)$ [10]. According to a recently published retrospective, cohort study involving 1452 patients with NAFLD and T2D, the risk of CVD among those patients was almost 70\% higher compared to patients with no liver disease $(H R=1.70$, $95 \% \mathrm{Cl}: 1.52-1.90)$ and was associated with a $60 \%$ greater risk of all-cause mortality [11].

However, it is worth mentioning that the observational design of the included studies in the aforemen- tioned meta-analyses does not permit safe conclusions to be drawn regarding the true causal relationship between NAFLD and CVD. It is still debated whether NAFLD represents a true predictor of CVD, while causality has to be proven in large-scale, prospective clinical studies [12].

Recent data suggest that liver fat content represents a risk factor for CVD [13]; however, proceeding a step further, the fibrosis stage seems to be the strongest predictor of both overall and disease-specific (including cardiovascular) mortality in patients with NAFLD/non-alcoholic steatohepatitis (NASH). Patients with liver fibrosis stage 3-4, irrespective of NAFLD activity score, exhibit the greatest risk of death among this population [14]. In general, obese/overweight patients feature greater risk of advanced liver fibrosis compared to lean patients with NAFLD, along with higher incidence rates of NASH [15]. Based on the increasing prevalence of overweight and obesity among patients with T2D, it seems that this association is becoming stronger.

A significant amount of interest lies on the pleiotropic effects of sodium glucose cotransporter 2 (SGLT-2) inhibitors. The hallmark EMPA-REG OUTCOME study revealed the potential role of empagliflozin in the management of patients with T2D at high cardiovascular risk, leading to a $38 \%$ decrease in the rates of cardiovascular death and a 35\% decrease in hospitalisation for heart failure [16].

In a post-hoc analysis of the EMPA-REG OUTCOME, it was shown that alanine aminotransferase (ALT) levels decreased from baseline to week 28 with empagliflozin compared to placebo (adjusted mean difference: -2.22 , $95 \% \mathrm{Cl}:-2.83,-1.62 ; p<0.0001)$ and remained signifi- 
cantly lower at week 164 (adjusted mean difference: $-1.26,95 \% \mathrm{Cl}:-2.12,-0.40 ; p=0.0040)$. Non-significant reduction in aspartate aminotransferase (AST) levels was observed at week 164 with empagliflozin vs. placebo. Alanine aminotransferase decrease was largely independent of alteration in body weight or glycaemic control [17]. The aforementioned results are consistent with empagliflozin-induced reduction in liver fat in patients with T2D. Of note, such a small decrease in ALT levels should be carefully interpreted regarding its clinical meaningfulness.

The recently published E-LIFT trial, enrolling 50 patients (42 completed the study) with T2D and NAFLD randomly assigned to empagliflozin $10 \mathrm{mg} /$ day as an add-on to standard antidiabetic treatment or placebo, demonstrated that empagliflozin led to a significant reduction in liver fat [18]. More specifically, empagliflozin resulted in a significant decrease in liver fat, by up to $4.0 \%$, compared to controls ( $p<0.0001)$, as measured by MRI-derived proton density fat fraction (MRI-PDFF), while a significant difference in liver fat, almost 5\%, was observed in the empagliflozin group in the end of the treatment $(16.2-11.3 \%, p<0.0001)$. Empagliflozin also produced a significant decrease in AST levels at the end of the treatment (64.3-49.7 IU/I, $p=0.001$ ), while it did not affect significantly ALT levels [18].

After meticulous interpretation of the results of the aforementioned studies [17, 18], along with the derived evidence documenting the cardiovascular efficacy and safety of empagliflozin [19, 20], we can speculate that empagliflozin promises to bridge the gap between NAFLD, T2D, and CVD, bolstering our armamentarium against this increasingly frequent concomitance that is associated with significant morbidity and mortality rates, especially in the Western world.

However, two certain limitations arise. First, there is no available randomised clinical trial assessing the efficacy of empagliflozin on overall and cardiovascular mortality, after detailed survival analysis, in patients with T2D and NAFLD/NASH. Secondarily, the effects of empagliflozin on AST/ALT levels and liver fat content should be cautiously interpreted, in terms of clinical significance. The efficacy of this novel agent on liver fibrosis amelioration should be evaluated in future, prospective clinical trials.

\section{Conflict of interest}

The authors declare no conflict of interest.

\section{References}

1. Byrne CD, Targher G. NAFLD: a multisystem disease. J Hepatol 2015; 62: S47-64
2. Saponaro C, Gaggini M, Gastaldelli A. Nonalcoholic fatty liver disease and type 2 diabetes: common pathophysiologic mechanisms. Curr Diab Rep 2015; 15: 607.

3. De Rosa S, Arcidiacono B, Chiefari E, et al. Type 2 diabetes mellitus and cardiovascular disease: genetic and epigenetic links. Front Endocrinol (Lausanne) 2018; 9: 2.

4. Zhou YY, Zhou XD, Wu SJ, et al. Nonalcoholic fatty liver disease contributes to subclinical atherosclerosis: a systematic review and meta-analysis. Hepatol Commun 2018; 2: 376-92.

5. Jaruvongvanich V, Chenbhanich J, Sanguankeo A, et al. Increased arterial stiffness in nonalcoholic fatty liver disease: a systematic review and meta-analysis. Eur J Gastroenterol Hepatol 2017; 29: e28-35.

6. Jaruvongvanich V, Wirunsawanya K, Sanguankeo A, Upala S. Nonalcoholic fatty liver disease is associated with coronary artery calcification: a systematic review and meta-analysis. Dig Liver Dis 2016; 48: 1410-7.

7. Madan SA, John F, Pyrsopoulos N, Pitchumoni CS. Nonalcoholic fatty liver disease and carotid artery atherosclerosis in children and adults: a meta-analysis. Eur J Gastroenterol Hepatol 2015; 27: 1237-48.

8. Fan Y, Wei F, Zhou Y, Zhang H. Association of non-alcoholic fatty liver disease with impaired endothelial function by flow-mediated dilation: a meta-analysis. Hepatol Res 2016; 46: E165-73.

9. Targher G, Byrne CD, Lonardo A, et al. Non-alcoholic fatty liver disease and risk of incident cardiovascular disease: a meta-analysis. J Hepatol 2016; 65: 589-600.

10. Mahfood Haddad T, Hamdeh S, Kanmanthareddy A, Alla VM. Nonalcoholic fatty liver disease and the risk of clinical cardiovascular events: a systematic review and meta-analysis. Diabetes Metab Syndr 2017; 11: S209-16.

11. Wild SH, Walker JJ, Morling JR, et al. Cardiovascular disease, cancer, and mortality among people with type 2 diabetes and alcoholic or nonalcoholic fatty liver disease hospital admission. Diabetes Care 2018; 41: 341-7.

12. Rinella ME. Nonalcoholic fatty liver disease: a systematic review. JAMA 2015; 313: 2263-73.

13. Lauridsen BK, Stender S, Kristensen TS, et al. Liver fat content, non-alcoholic fatty liver disease, and ischaemic heart disease: mendelian randomization and meta-analysis of 279013 individuals. Eur Heart J 2018; 39: 385-93.

14. Ekstedt $M$, Hagström $H$, Nasr P, et al. Fibrosis stage is the strongest predictor for disease-specific mortality in NAFLD after up to 33 years of follow-up. Hepatology 2015; 61: 1547-54.

15. Sookoian S, Pirola CJ. Systematic review with meta-analysis: the significance of histological disease severity in lean patients with nonalcoholic fatty liver disease. Aliment Pharmacol Ther 2018; 47: 16-25.

16. Zinman B, Wanner C, Lachin JM, et al. Empagliflozin, cardiovascular outcomes, and mortality in type 2 diabetes. $N$ Engl J Med 2015; 373: 2117-28.

17. Sattar N, Fitchett D, Hantel S, et al. Empagliflozin is associated with improvements in liver enzymes potentially consistent with reductions in liver fat: results from randomised trials including the EMPA-REG OUTCOME® trial. Diabetologia 2018; 61: 2155-63.

18. Kuchay MS, Krishan S, Mishra SK, et al. Effect of empagliflozin on liver fat in patients with type 2 diabetes and nonalcoholic 
fatty liver disease: a randomized controlled trial (E-LIFT trial). Diabetes Care 2018; 41: 1801-8.

19. Salsali A, Kim G, Woerle HJ, et al. Cardiovascular safety of empagliflozin in patients with type 2 diabetes: a meta-analysis of data from randomized placebo-controlled trials. Diabetes Obes Metab 2016; 18: 1034-40.

20. Zheng SL, Roddick AJ, Aghar-Jaffar R, et al. Association between use of sodium-glucose cotransporter 2 inhibitors, glucagon-like peptide 1 agonists, and dipeptidyl peptidase 4 inhibitors with all-cause mortality in patients with type 2 diabetes: a systematic review and meta-analysis. JAMA 2018; 319: 1580-91.

Received: 24.08 .2018

Accepted: 27.09.2018 